\title{
The Growth Response of Several Potato Genotypes (Solanum tuberosum L.) to Induced Water Stress Using Sorbitol and Polyethylene Glycol
}

\author{
Mihaela A. CIOLOCA*, Andreea M. TICAN, Maria IANOŞI, \\ Carmen L. BĂDĂRĂU
}

National Institute of Research and Development for Potato and Sugar Beet, Research Laboratory for Plant Tissue Culture, 2 Fundäturii Street, 500470,Brasov,Romania; mihaela.cioloca@potato.ro ("corresponding author)

\begin{abstract}
The current paper aimed to study the in vitro response of potato genotypes to water stress induced by adding sorbitol and polyethylene glycol in the culture medium. The biological material analysed in the experiment was represented by a Romanian line 'LP 11-1525/1' and two isogenic lines 'LI 101' and 'LI 102'. For cultures initiation, the line 'LP 11-1525/1' was started from meristems and for the other two genotypes true potato seeds were used. The studied potato genotypes behaved differently depending on the analysed parameters and on the treatment applied for drought tolerance. It was noted that the line 'LP 111525/1' achieved good results for most of the growth parameters studied, and also the lines derived from true potato seeds behaved well, in some cases even exceeding the line derived from meristems. Of the lines derived from true potato seeds, the best performance was noted for line 'LI 101-6' in all the analysed parameters, both on sorbitol and PEG medium. In addition, lines 'LI 101-7' and 'LI 102-4' achieved good results on both variants of medium used to mediate water stress. Therefore, establishing drought tolerance individuals within populations derived from true potato seeds using sorbitol and polyethylene glycol might be applied.
\end{abstract}

Keywords: drought, in vitro, Solanum tuberosum, stress, true potato seed

\section{Introduction}

Potato (Solanum tuberosum L.) is one of the most important vegetables in the world (Albiski et al., 2012). There are currently numerous potato varieties that are adapted to diverse environments with different soil types and climates (Barra et al., 2013). According to the FAO, these characteristics lead the crop to play an important role in world food security (FAO, 2011, cited by Barra, 2013).

The identification of new potato genotypes tolerant to abiotic stress is currently needed since climate change is associated with an increase in temperature of the planet and a decrease in precipitation (Lobell et al., 2011, cited by Barra, 2013).

In the past years Romania was also faced with droughts which affected crop species, including potato, especially when drought was installed in the early stages of the growing season and was extended for a longer time. In this context, Romanian researchers work was oriented to create new potato varieties tolerant to adverse environmental conditions.

High temperatures and lack of rain are abiotic stresses that cause significant decreases in potato production (Arvin and Donnelly, 2008). Abiotic stress refers to the negative impact of environmental factors on plant growth and development. Among these factors it can be mention: extreme temperatures, flooding, high winds, drought, fire, stoniness ground, pollution, soil acidity etc. (Marron et al., 2008). These factors are unavoidable and when they are pulled together cause even greater damages (Mittler, 2006). For example, crop plants are largely dependent on the availability of moisture in the top 10 $\mathrm{cm}$ of the soil profile. Drought stress occurs when soil moisture status is low, relative humidity is low and temperature is high. If the drought persists, plants dry up, and productivity of crops gets badly affected (Pareek et al., 2010). Salinity is also considered as a major abiotic stress and significantly affecting crop production all over the world, especially in arid and semiarid regions (Khajeh-Hosseini et al., 2003). Various physiological injuries have been observed under high temperatures, such as scorching of leaves and stems, leaf abscission and senescence, shoot and root growth inhibition or fruit damage (Vollenweider and Günthardt-Goerg, 2005).

In regard of this, the potato production is adjusted towards new regions of the world where high temperatures and limited water resources, or both, are restrictive factors for achieving quality productions, but also a challenging task for researchers is to find new potato genotypes tolerant of those limiting factors mentioned above (Levy and Veilleux, 2007). 
512

The drought is a major environmental factor that determines the plant growth, the yield and the distribution of plants (Rukundo et al., 2012). Currently, the drought represents the most worldwide crop reducing stress in agriculture (Ober, 2008 cited by Rukundo, 2012). The drought affects more than 10\% of arable soil (Bray et al., 2000; Zidenga, 2006, cited by Rukundo, 2012) and continue to increase, due to the explosive expansion of world population, continuous deterioration of arable land, shortage of fresh water, and the current climate change.

The increase in drought stress threatens the global agriculture production and food availability (Rokundo et al., 2012). It has been estimated that two thirds of the yield potential of major crops are routinely lost due to drought stress (Bray et al., 2000; Lafitte et al., 2004; Zidenga, 2006; Magombeyi and Taigbenu, 2008, cited by Rukundo, 2012). Therefore, the sustainability of production will depend on the identification and development of new drought tolerant varieties (Cochard et al., 2008, cited by Rukundo, 2012).

Polyethylene glycol (PEG) is a polymer produced in a range of molecular weights. PEG of higher molecular weight $(4,000$ to 8,000 ) was commonly used in physiological experiments to induce controlled drought stress in nutrient solution cultures (Hassanpanah, 2009). Polyethylene glycol (PEG), sucrose, mannitol or sorbitol has been used by several researches as osmotic stress agents for in vitro selection (Hassan et al., 2004). However, PEG has been the most extensively used to stimulate water stress in plants. This compound of high molecular weight is a non-penetrating inert osmoticum that reduces water potential of nutrient solutions without being taken up by the plant or being phytotoxic (Hassan et al., 2004).

Sorbitol, a six carbon sugar alcohol, is one of the most frequently found polyols in plants. It is a direct product of photosynthesis in mature leaves, in parallel with sucrose, whereas both serve similar functions, such as translocation of carbon skeletons and energy between sources and sink organs (Jain et al., 2010). Increased transport of polyols, both in the xylem and phloem, occurs frequently as a result of salt or drought stress (Noiraud et al., 2001).

The purpose of the present study was to determine the response to water stress of nine potato genotypes, by measuring the morphological characteristics associated with the vegetative growth of potato plantlets in vitro using PEG and sorbitol as a water stress inductors. In order to achieve high productions and biological quality for improved potato crops, it is important to produce local potato varieties tolerant to drought. In the hereby study it was tried the identification of potato genotypes tolerant to water stress, obtained both through vegetative (meristems) and generative (true potato seeds) propagation.

\section{Materials and Methods}

\section{Plantmaterial}

The study was carried out at the National Institute of Research and Development for Potato and Sugar Beet Brasov (Romania), Research Laboratory for Plant Tissue Culture. The biological material analysed in the current experiment was represented by a Romanian line 'LP 11-1525/1' and two isogenic lines from Dutch company Bejo Zaden: 'LI 101' and 'LI 102'.
The line 'LP 11-1525/1' was started from meristems for cultures initiation, and for the other two genotypes true potato seeds were used. In the case of isogenic lines, were selected for the study those individuals who have a favourable outcome in a medium supplemented with $15 \mathrm{~g} / \mathrm{L}$ mannitol. The line 'LP 11$1525 / 1$ ' seedlings were grown on the Murashige-Skoog medium.

All the materials were checked for the PVX (potato virus X), PVS (potato virus S), PVY (potato virus Y) and PVM (potato virus $\mathrm{M}$ ). After ELISA testing, results showed that the plants were healthy.

\section{Water stress tolerance and growth parameters}

To multiply and test water stress tolerance in vitro, there were used $1 \mathrm{~cm}$ long explants containing an axillary bud with related leaf and half of neighbouring internodes. After inoculation, the cultures were incubated in a growth chamber (temperature $20 \pm 2{ }^{\circ} \mathrm{C}, 16: 8$ photoperiod) for 6 weeks. During this period a series of observations and notations were made at 2, 4, and 6 weeks on the following parameters: stem length, number of leaves, leaf aspect, rooting, plantlet fresh and dry weight, root length, fresh and dry root weight.

Within the observations made at 2 and 4 weeks of in vitro culture for leaf aspect assessment a grading scale with seven levels was used, as follows: 1 - very small; 2 - very small - small; 3 - small; 4 - small - medium; 5 - medium; 6 - medium large; 7 - large.

Observations concerning the level of rooting was done similarly to the method of leaf aspect assessment and for this purpose a rating scale with values ranging from 1 to 5 was used as follows: 1 - no roots; 2 - very poorly developed roots; 3 weakly developed roots; 4 - well developed roots.

In order to determine the response of the potato plantlets to artificially induced water stress conditions, three different variants were used: Murashige-Skoog (MS) added with $48 \mathrm{~g} / \mathrm{L}$ PEG, MS medium added with $40 \mathrm{~g} / \mathrm{L}$ sorbitol and MS medium containing $20 \mathrm{~g} / \mathrm{L}$ sucrose, as a control. For solidification of all three variants, $9 \mathrm{~g} / \mathrm{L}$ agar was used.

The $\mathrm{pH}$ of the solutions was adjusted at 5.7 with a $\mathrm{pH}$ meter. About $5 \mathrm{ml}$ of nutrient medium was distributed in tubes of $2 \mathrm{~cm}$ diameter and $15 \mathrm{~cm}$ height, which were covered with aluminium foil caps.

The sterilization was carried out in the autoclave at $121^{\circ} \mathrm{C}$ for 20 minutes. After sterilization, the medium was removed from the autoclave and allowed to cool and solidify until the next day when inoculation of stem explants has been started.

\section{Statistical analyses}

The study was design as a randomized complete block with three replicates; factors included 3 potato genotypes ('LI 101', 'LI 102' and 'LP 11-1525/1'), 3 variants of medium (MS supplement with $40 \mathrm{~g} / \mathrm{L}$ sorbitol, MS supplement with $48 \mathrm{~g} / \mathrm{L}$ PEG and MS as control) and three measurement times (2, 4 and 6 weeks).

The data were statistically analysed using SPSS program. The response of each potato genotype to PEG and sorbitol induced water stress was measured for each morphological parameter and its value under stress conditions was related to its respective control, in three different periods of time. The statistical analysis was performed by comparing the means, specifying standard deviation. For comparing the obtained means, ANOVA procedure and Duncan's multiple range test 
was applied. Correlation between all the characters has been made. Correlation of measured parameters was performed using Pearson correlation.

\section{Results and Discussion}

Regarding the stem length of the studied potato genotypes, Duncan test analysis indicated close values, thus no significant differences in the medium with sorbitol or PEG (Table 1). Throughout observations, water stress caused a decrease in stem length of all lines. However, the lines 'LI 101-6' and 'LI 101-7' have achieved good and consistent results for all the time periods, both on sorbitol and PEG medium (Table 1).
Table 2 presents the behaviour of potato genotypes regarding the number of leaves. Several lines results on medium added with sorbitol and PEG were higher or similar to those obtained on control. Lines 'LI 101-6', 'LI 101-7' and 'LI 102-4' have achieved good and consistent results both on sorbitol and PEG medium (Table 2).

Table 3 shows the response of potato genotypes regarding the leaf aspect. Throughout observations, leaf aspect decreased due to drought in all lines. However, the lines LI '101-6' and 'LI 101-7' have achieved good and consistent results both on sorbitol and PEG medium (Table 3).

Table 1. Stem length of the studied potato genotypes under induced water stress

\begin{tabular}{|c|c|c|c|c|c|c|c|c|}
\hline \multirow{2}{*}{ Line (cod) } & \multicolumn{6}{|c|}{ Stem length $(\mathrm{cm})$} & \multicolumn{2}{|c|}{ Relative response compared to the control (\% } \\
\hline & \multicolumn{2}{|c|}{ Control } & \multicolumn{2}{|c|}{ Sorbitol $4 \%$} & \multicolumn{2}{|c|}{ PEG $4.8 \%$} & \multirow[t]{2}{*}{ Sorbitol $4 \%$} & \multirow[t]{2}{*}{ PEG $4.8 \%$} \\
\hline \multicolumn{7}{|c|}{2 weeks } & & \\
\hline 'LI 101-3' & 3.62 & bcd & 0.52 & $\mathrm{f}$ & 0.80 & $\mathrm{f}$ & 14.4 & 22.1 \\
\hline 'LI 101-6' & 3.46 & bcd & 0.60 & $\mathrm{f}$ & 0.78 & $\mathrm{f}$ & 17.3 & 22.5 \\
\hline 'LI 101-7’ & 2.80 & cde & 0.44 & $\mathrm{f}$ & 0.72 & $\mathrm{f}$ & 15.7 & 25.7 \\
\hline 'LI 102-2' & 2.24 & def & 0.54 & $\mathrm{f}$ & 0.88 & $\mathrm{f}$ & 24.1 & 39.3 \\
\hline 'LI 102-3' & 4.90 & $\mathrm{ab}$ & 0.80 & $\mathrm{f}$ & 0.30 & $\mathrm{f}$ & 16.3 & 6.1 \\
\hline 'LI 102-4' & 4.12 & $a b c$ & 0.78 & $\mathrm{f}$ & 0.46 & $\mathrm{f}$ & 18.9 & 11.2 \\
\hline 'LP 11-1525/1-1' & 5.20 & $\mathrm{ab}$ & 0.44 & $\mathrm{f}$ & 0.68 & $\mathrm{f}$ & 8.5 & 13.1 \\
\hline 'LP 11-1525/1-2' & 5.88 & $\mathrm{a}$ & 0.66 & f & 1.12 & ef & 11.2 & 19.1 \\
\hline 'LP 11-1525/1-3' & 5.68 & a & 1.08 & ef & 0.62 & $\mathrm{f}$ & 19.0 & 10.9 \\
\hline \multicolumn{9}{|c|}{4 weeks } \\
\hline 'LI 101-3' & 9.00 & $\mathrm{ab}$ & 1.04 & c & 1.34 & c & 11.6 & 14.9 \\
\hline 'LI 101-6' & 7.44 & $\mathrm{~b}$ & 1.54 & c & 1.40 & c & 20.7 & 18.8 \\
\hline 'LI 101-7’ & 7.52 & $\mathrm{~b}$ & 1.54 & c & 1.62 & c & 20.5 & 21.5 \\
\hline 'LI 102-2' & 8.20 & $\mathrm{ab}$ & 0.60 & c & 2.76 & c & 7.3 & 33.7 \\
\hline 'LI 102-3' & 10.80 & a & 2.62 & c & 0.56 & c & 24.3 & 5.2 \\
\hline 'LI 102-4' & 8.48 & $\mathrm{ab}$ & 1.06 & c & 0.92 & c & 12.5 & 10.9 \\
\hline 'LP 11-1525/1-1' & 7.50 & $\mathrm{~b}$ & 1.78 & c & 2.30 & c & 23.7 & 30.7 \\
\hline 'LP 11-1525/1-2' & 8.02 & $\mathrm{ab}$ & 1.88 & c & 3.02 & c & 23.4 & 37.7 \\
\hline 'LP 11-1525/1-3' & 9.38 & $\mathrm{ab}$ & 2.10 & c & 2.20 & c & 22.4 & 23.5 \\
\hline \multicolumn{9}{|c|}{6 weeks } \\
\hline 'LI 101-3' & 13.94 & $\mathrm{ab}$ & 1.72 & d & 1.38 & d & 12.3 & 9.9 \\
\hline 'LI 101-6' & 9.12 & c & 2.62 & d & 2.22 & d & 28.7 & 24.3 \\
\hline 'LI 101-7’ & 12.62 & $\mathrm{ab}$ & 2.54 & d & 1.72 & d & 20.1 & 13.6 \\
\hline 'LI 102-2' & 13.58 & $\mathrm{ab}$ & 0.76 & d & 3.80 & d & 5.6 & 28.0 \\
\hline 'LI 102-3' & 15.66 & a & 4.46 & d & 1.08 & d & 28.5 & 6.9 \\
\hline 'LI 102-4' & 11.56 & $\mathrm{bc}$ & 1.54 & d & 1.18 & d & 13.3 & 10.2 \\
\hline 'LP 11-1525/1-1' & 8.38 & c & 2.12 & d & 3.30 & d & 25.3 & 39.4 \\
\hline 'LP 11-1525/1-2' & 8.98 & c & 2.20 & d & 3.50 & d & 24.5 & 39.0 \\
\hline 'LP 11-1525/1-3' & 10.58 & $\mathrm{bc}$ & 2.80 & d & 3.12 & d & 26.5 & 29.5 \\
\hline \multicolumn{9}{|c|}{ Mean } \\
\hline 2 weeks & 4.21 & a & 0.65 & $\mathrm{~b}$ & 0.70 & $\mathrm{~b}$ & $16.2 \pm 4.6$ & $18.9 \pm 10.0$ \\
\hline 4 weeks & 8.48 & $\mathrm{a}$ & 1.57 & $\mathrm{~b}$ & 1.79 & $\mathrm{~b}$ & $18.5 \pm 6.3$ & $21.9 \pm 10.8$ \\
\hline 6 weeks & 11.60 & $\mathrm{a}$ & 2.31 & b & 2.37 & b & $20.5 \pm 8.3$ & $22.3 \pm 12.6$ \\
\hline
\end{tabular}

Different letters indicate statistically significant differences between different treatments. (Duncan's multiple range test; $\mathrm{P}<0.05$ ) 
Table 2. The number of leaves of the studied potato genotypes under induced water stress

\begin{tabular}{|c|c|c|c|c|c|c|c|c|c|}
\hline \multirow{2}{*}{ Line (cod) } & \multicolumn{7}{|c|}{ Number of leaves } & \multicolumn{2}{|c|}{ Relative response compared to the control (\% } \\
\hline & \multicolumn{2}{|c|}{ Control } & \multicolumn{2}{|c|}{ Sorbitol $4 \%$} & \multicolumn{3}{|c|}{ PEG $4.8 \%$} & \multirow[t]{2}{*}{ Sorbitol $4 \%$} & \multirow[t]{2}{*}{ PEG $4.8 \%$} \\
\hline \multicolumn{8}{|c|}{2 weeks } & & \\
\hline 'LI 101-3' & 6.00 & a & 2.60 & & & 3.80 & abcde & 43.3 & 63.3 \\
\hline 'LI 101-6' & 4.20 & abcde & 3.00 & $\mathrm{cc}$ & & 4.20 & abcde & 71.4 & 100.0 \\
\hline 'LI 101-7’ & 5.80 & $\mathrm{ab}$ & 4.20 & $\mathrm{ab}$ & & 3.80 & abcde & 72.4 & 65.5 \\
\hline 'LI 102-2' & 4.00 & abcde & 1.20 & & & 3.80 & abcde & 30.0 & 95.0 \\
\hline 'LI 102-3' & 5.40 & $a b c$ & 3.40 & bc & & 2.40 & ef & 63.0 & 44.4 \\
\hline 'LI 102-4' & 4.40 & abcde & 4.40 & $\mathrm{ab}$ & & 3.20 & cdef & 100.0 & 72.7 \\
\hline 'LP 11-1525/1-1' & 5.00 & $a b c d$ & 3.00 & & & 3.40 & bcdef & 60.0 & 68.0 \\
\hline 'LP 11-1525/1-2' & 5.80 & $a b$ & 3.60 & $\mathrm{abc}$ & & 4.00 & abcde & 62.1 & 69.0 \\
\hline 'LP 11-1525/1-3' & 5.80 & $\mathrm{ab}$ & 4.40 & $\mathrm{ab}$ & & 3.40 & bcdef & 75.9 & 58.6 \\
\hline \multicolumn{10}{|c|}{4 weeks } \\
\hline 'LI 101-3' & 9.20 & $\mathrm{ab}$ & 4.60 & ef & & 5.20 & cdefgh & 50.0 & 56.5 \\
\hline 'LI 101-6’ & 6.40 & abcdefg & 4.20 & $f_{\xi}$ & & 6.00 & bcdefg & 65.6 & 93.8 \\
\hline ‘LI 101-7’ & 9.80 & a & 6.80 & $a b c$ & & 5.00 & defgh & 69.4 & 51.0 \\
\hline 'LI 102-2' & 8.00 & abcde & 2.40 & 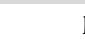 & & 5.80 & bcdefg & 30.0 & 72.5 \\
\hline 'LI 102-3' & 8.00 & abcde & 6.20 & $\mathrm{bcc}$ & & 3.80 & gh & 77.5 & 47.5 \\
\hline 'LI 102-4' & 6.80 & abcdefg & 5.20 & $\mathrm{cdc}$ & & 4.60 & efgh & 76.5 & 67.7 \\
\hline 'LP 11-1525/1-1' & 8.20 & abcd & 7.80 & $a b$ & & 6.00 & bcdefg & 95.1 & 73.2 \\
\hline 'LP 11-1525/1-2' & 9.00 & $\mathrm{ab}$ & 7.00 & $a b c$ & & 7.40 & abcdef & 77.9 & 83.2 \\
\hline 'LP 11-1525/1-3' & 8.60 & $a b c$ & 7.20 & $a b c$ & & 5.40 & cdefgh & 83.7 & 62.8 \\
\hline \multicolumn{10}{|c|}{6 weeks } \\
\hline 'LI 101-3' & 9.60 & $a b c$ & 6.00 & cdef & 8.20 & & abcde & 62.5 & 85.4 \\
\hline 'LI 101-6' & 6.00 & cdef & 5.60 & def & 7.40 & & abcdef & 93.3 & 123.3 \\
\hline 'LI 101-7’ & 10.20 & a & 8.60 & abcde & 6.20 & & bcdef & 84.3 & 60.8 \\
\hline 'LI 102-2' & 9.80 & $a b c$ & 3.60 & $\mathrm{f}$ & 7.80 & & abcde & 36.7 & 79.6 \\
\hline 'LI 102-3' & 9.80 & $a b c$ & 8.00 & abcde & 5.40 & & ef & 81.6 & 55.1 \\
\hline 'LI 102-4' & 9.20 & abcde & 7.20 & abcdef & 7.20 & & abcdef & 78.3 & 78.3 \\
\hline 'LP 11-1525/1-1' & 10.00 & $a b$ & 8.20 & abcde & 7.40 & & abcdef & 82.0 & 74.0 \\
\hline 'LP 11-1525/1-2' & 10.40 & a & 9.20 & abcde & 8.20 & & abcde & 88.5 & 78.9 \\
\hline 'LP 11-1525/1-3' & 10.20 & a & 9.40 & $a b c d$ & 7.40 & & abcdef & 92.2 & 72.5 \\
\hline \multicolumn{10}{|c|}{ Mean } \\
\hline 2 weeks & 5.16 & a & 3.31 & $\mathrm{~b}$ & 3.56 & & $\mathrm{~b}$ & $64.2 \pm 19.9$ & $70.7 \pm 17.3$ \\
\hline 4 weeks & 8.22 & a & 5.71 & $\mathrm{~b}$ & 5.47 & & $\mathrm{~b}$ & $69.5 \pm 19.4$ & $67.5 \pm 14.9$ \\
\hline 6 weeks & 9.47 & a & 7.31 & b & 7.24 & & $\mathrm{~b}$ & $77.7 \pm 17.9$ & $78.7 \pm 19.3$ \\
\hline
\end{tabular}

Different letters indicate statistically significant differences between different treatments. (Duncan's multiple range test; $\mathrm{P}<0.05$ )

The response of potato genotypes regarding the level of rooting at 2 and 4 weeks, respectively roots length at 6 weeks was illustrated in Table 4 . After 2 and 4 weeks drought caused a decrease in level of rooting of all lines. Regarding the roots length, after 6 weeks some lines have achieved better results on the sorbitol medium than control. Thus, in line 'LI 101-3', 'LP 11-1525/1-1', 'LP 11-1525/1-2' and 'LP 11-1525/1-3' the sorbitol treatment resulted in roots length increases (Table 4). Line 'LI 101-6' achieved good results for all time periods both on sorbitol and PEG medium (Table 4).

After 6 weeks of in vitro cultivation of potato plantlets in addition to parameters measured at 2 and 4 weeks, other determinations were carried out. Table 5 shows the behaviour of potato genotypes regarding the total plant fresh weight. Induced water stress caused a decrease in fresh weight of all lines (Table 5). However, line 'LI 101-6' has achieved good results both under sorbitol and PEG treatments (Table 5).

Except line 'LI 102-3' which obtained a higher value under sorbitol treatment $(0.017 \mathrm{~g})$ and the line 'LI 102-2' which obtained the same amount both under PEG treatment as the control $(0.015 \mathrm{~g})$, induced water stress factors determined a lower amount regarding the total dry weight compared to the control (Table 6). Lines 'LI 102-4' and 'LI 101-6' have achieved good results both under sorbitol and PEG treatments (Table 6). 
Table 3. Leaf aspect of the studied potato genotypes under induced water stress

\begin{tabular}{|c|c|c|c|c|c|c|c|c|}
\hline \multirow{2}{*}{ Line (cod) } & \multicolumn{6}{|c|}{ Leaf aspect (scale) } & \multicolumn{2}{|c|}{ Relative response compared to the control (\%) } \\
\hline & \multicolumn{2}{|c|}{ Control } & \multicolumn{2}{|c|}{ Sorbitol 4\% } & \multicolumn{2}{|c|}{ PEG $4.8 \%$} & \multirow[t]{2}{*}{ Sorbitol $4 \%$} & \multirow[t]{2}{*}{ PEG 4.8\% } \\
\hline \multicolumn{7}{|c|}{2 weeks } & & \\
\hline 'LI 101-3' & 5.0 & a & 1.0 & ef & 1.4 & ef & 20.0 & 28.0 \\
\hline 'LI 101-6' & 3.0 & abcdef & 2.0 & def & 1.8 & ef & 66.7 & 60.0 \\
\hline 'LI 101-7’ & 5.0 & a & 2.4 & bcdef & 2.2 & cdef & 48.0 & 44.0 \\
\hline 'LI 102-2' & 2.4 & bcdef & 0.6 & $\mathrm{f}$ & 1.2 & ef & 25.0 & 50.0 \\
\hline 'LI 102-3' & 3.4 & abcde & 1.4 & ef & 1.0 & ef & 41.2 & 29.4 \\
\hline 'LI 102-4' & 3.2 & abcde & 2.2 & cdef & 1.0 & ef & 68.8 & 31.3 \\
\hline 'LP 11-1525/1-1' & 4.4 & abcd & 2.0 & def & 2.0 & def & 45.5 & 45.5 \\
\hline 'LP 11-1525/1-2’ & 4.6 & $a b c$ & 2.0 & def & 3.0 & abcdef & 43.5 & 65.2 \\
\hline 'LP 11-1525/1-3' & 4.8 & $\mathrm{ab}$ & 3.0 & abcdef & 2.0 & def & 62.5 & 41.7 \\
\hline \multicolumn{9}{|c|}{4 weeks } \\
\hline 'LI 101-3' & 5.0 & $a b c$ & 2.2 & efgh & 1.4 & fgh & 44.0 & 28.0 \\
\hline 'LI 101-6' & 3.0 & cdefgh & 2.2 & efgh & 1.6 & fgh & 73.3 & 53.3 \\
\hline ‘LI 101-7’ & 5.0 & $a b c$ & 3.0 & cdefg & 3.4 & bcdef & 60.0 & 68.0 \\
\hline 'LI 102-2' & 4.2 & abcde & 0.6 & $\mathrm{~h}$ & 1.2 & gh & 14.3 & 28.6 \\
\hline 'LI 102-3' & 4.2 & abcde & 2.6 & defgh & 1.2 & gh & 61.9 & 28.6 \\
\hline 'LI 102-4' & 4.6 & abcd & 3.0 & cdefg & 1.4 & fgh & 65.2 & 30.4 \\
\hline 'LP 11-1525/1-1' & 5.0 & $a b c$ & 4.4 & abcd & 3.0 & cdefg & 88.0 & 60.0 \\
\hline 'LP 11-1525/1-2' & 5.4 & $\mathrm{ab}$ & 4.2 & abcde & 3.4 & bcdef & 77.8 & 63.0 \\
\hline 'LP 11-1525/1-3' & 6.0 & a & 5.0 & $a b c$ & 3.2 & cdefg & 83.3 & 53.3 \\
\hline \multicolumn{9}{|c|}{6 weeks } \\
\hline 'LI 101-3' & 5.8 & abcd & 1.8 & $\mathrm{ij}$ & 1.8 & ij & 31.0 & 31.0 \\
\hline 'LI 101-6' & 3.0 & fghi & 2.8 & ghij & 1.8 & $\mathrm{ij}$ & 93.3 & 60.0 \\
\hline 'LI 101-7’ & 6.4 & $\mathrm{ab}$ & 4.4 & cdefg & 3.2 & efghi & 68.8 & 50.0 \\
\hline 'LI 102-2' & 5.0 & abcde & 1.0 & j & 2.6 & ghij & 20.0 & 52.0 \\
\hline 'LI 102-3' & 4.8 & bcdef & 4.2 & defg & 1.4 & ij & 87.5 & 29.2 \\
\hline 'LI 102-4' & 6.8 & a & 3.0 & fghi & 2.2 & hij & 44.2 & 32.4 \\
\hline 'LP 11-1525/1-1' & 6.2 & $a b c$ & 4.0 & defgh & 2.6 & ghij & 64.5 & 41.9 \\
\hline 'LP 11-1525/1-2' & 5.8 & abcd & 4.0 & defgh & 3.0 & fghi & 69.0 & 51.7 \\
\hline 'LP 11-1525/1-3' & 5.8 & abcd & 4.0 & defgh & 2.6 & ghij & 69.0 & 44.8 \\
\hline \multicolumn{9}{|c|}{ Mean } \\
\hline 2 weeks (scale) ${ }^{*}$ & 3.98 & a & 1.84 & $\mathrm{~b}$ & 1.73 & $\mathrm{~b}$ & $46.8 \pm 17.2$ & $43.9 \pm 13.1$ \\
\hline 4 weeks (scale) & 4.71 & a & 3.02 & $\mathrm{~b}$ & 2.20 & c & $63.1 \pm 22.7$ & $45.9 \pm 16.8$ \\
\hline 6 weeks (scale) & 5.51 & $\mathrm{a}$ & 3.24 & b & 2.36 & c & $60.8 \pm 24.5$ & $43.7 \pm 10.9$ \\
\hline
\end{tabular}

Different letters indicate statistically significant differences between different treatments. (Duncan's multiple range test; $\mathrm{P}<0.05$ ).

${ }^{*}$ Leaf aspect: scale from 1 (very small leaves) to 7 (large leaves)

Regarding the treatment for inducing drought in laboratory conditions, the average values of the characteristics indicated that PEG had a stronger effect than sorbitol, except for stem length. The obtained results showed that some lines had higher values on sorbitol medium than PEG one, while others had higher values on PEG medium than sorbitol ones. Thus, for all time periods and for all measured parameters the line 'LI 102-2' obtained better results on PEG medium than sorbitol one and the line 'LI 102-3' perform better on sorbitol than PEG one. Also, lines 'LI 101-3' and 'LI 102-4' obtained generally better results on sorbitol medium than PEG one.
Regarding the correlations between morphological characteristics under induced water stress, the values were significant for all the parameters (Table 7).

\section{Discussion}

A good approach to evaluate water stress tolerance is with field trials, but environmental variables are difficult to control (Barra et al., 2013). An alternative to minimize this effect is to develop in vitro assays (Gopal et al., 2008; Rahman et al., 2008). Various in vitro methods to induce water stress in plants have been described, which use 
Table 4. Level of rooting and roots length of the studied potato genotypes under induced water stress

\begin{tabular}{|c|c|c|c|c|c|c|c|c|}
\hline \multirow{2}{*}{ Line (cod) } & \multicolumn{6}{|c|}{ Level of rooting (scale) } & \multicolumn{2}{|c|}{$\begin{array}{l}\text { Relative response compared to the control } \\
\qquad(\%)\end{array}$} \\
\hline & \multicolumn{2}{|c|}{ Control } & \multicolumn{2}{|c|}{ Sorbitol 4\% } & \multicolumn{2}{|c|}{ PEG $4.8 \%$} & Sorbitol 4\% & PEG $4.8 \%$ \\
\hline \multicolumn{9}{|c|}{2 weeks } \\
\hline 'LI 101-3' & 4.4 & a & 2.8 & cdefgh & 1.7 & ghi & 63.6 & 38.6 \\
\hline 'LI 101-6’ & 3.4 & abcde & 2.6 & defghi & 2.0 & efghi & 76.5 & 58.8 \\
\hline ‘LI 101-7’ & 4.2 & $a b c$ & 2.0 & efghi & 1.6 & ghi & 47.6 & 38.1 \\
\hline 'LI 102-2’' & 3.4 & abcde & 1.2 & $\mathrm{i}$ & 1.8 & fghi & 35.3 & 52.9 \\
\hline 'LI 102-3’ & 3.5 & abcd & 2.0 & efghi & 1.4 & hi & 57.1 & 40.0 \\
\hline 'LI 102-4' & 3.2 & abcdef & 1.8 & fghi & 1.4 & hi & 56.3 & 43.8 \\
\hline 'LP 11-1525/1-1' & 4.4 & a & 2.9 & bcdefg & 1.9 & fghi & 65.9 & 43.2 \\
\hline 'LP 11-1525/1-2' & 4.3 & $\mathrm{ab}$ & 2.7 & defgh & 2.3 & defghi & 62.8 & 53.5 \\
\hline 'LP 11-1525/1-3’ & 4.6 & a & 3.7 & abcd & 2.3 & defghi & 80.4 & 50.0 \\
\hline \multicolumn{9}{|c|}{4 weeks } \\
\hline 'LI 101-3’ & 5.0 & $a b c$ & 3.5 & bcdefg & 2.2 & ghij & 70.0 & 44.0 \\
\hline 'LI 101-6’ & 3.0 & cdefgh & 2.6 & efghij & 2.5 & efghij & 68.4 & 65.8 \\
\hline 'LI 101-7’ & 5.0 & $a b c$ & 2.5 & efghij & 2.7 & efghij & 50.0 & 54.0 \\
\hline 'LI 102-2' & 4.2 & abcde & 1.4 & j & 2.0 & hij & 35.9 & 51.3 \\
\hline 'LI 102-3’’ & 4.2 & abcde & 2.8 & defghij & 1.5 & ij & 65.1 & 34.9 \\
\hline 'LI 102-4' & 4.6 & abcd & 2.2 & ghij & 2.2 & ghij & 53.7 & 53.7 \\
\hline 'LP 11-1525/1-1' & 5.0 & $a b c$ & 4.2 & $\mathrm{abcd}$ & 2.4 & fghij & 89.4 & 51.1 \\
\hline 'LP 11-1525/1-2’ & 5.4 & $\mathrm{ab}$ & 3.9 & abcde & 3.1 & cdefgh & 84.8 & 67.4 \\
\hline 'LP 11-1525/1-3’ & 6.0 & $\mathrm{a}$ & 4.2 & abcd & 2.9 & cdefghi & 87.5 & 60.4 \\
\hline \multicolumn{9}{|c|}{ Root length (cm) } \\
\hline \multicolumn{9}{|c|}{6 weeks } \\
\hline 'LI 101-3’ & 10.76 & $\mathrm{ab}$ & 11.04 & $\mathrm{ab}$ & 3.82 & defg & 102.6 & 35.5 \\
\hline 'LI 101-6’ & 6.60 & abcdefg & 5.76 & bcdefg & 4.08 & cdefg & 87.3 & 61.8 \\
\hline 'LI 101-7’ & 11.66 & a & 6.20 & bcdefg & 4.06 & cdefg & 53.2 & 34.8 \\
\hline 'LI 102-2' & 9.42 & $a b$ & 3.24 & efg & 3.28 & efg & 34.4 & 34.8 \\
\hline 'LI 102-3’ & 8.20 & abcde & 7.12 & abcdef & 1.48 & $\mathrm{~g}$ & 86.8 & 18.1 \\
\hline 'LI 102-4' & 9.64 & $\mathrm{ab}$ & 3.38 & efg & 2.60 & $\mathrm{fg}$ & 35.1 & 27.0 \\
\hline 'LP 11-1525/1-1' & 9.26 & $\mathrm{ab}$ & 9.60 & $\mathrm{ab}$ & 2.98 & efg & 103.7 & 32.2 \\
\hline 'LP 11-1525/1-2' & 8.90 & abcd & 9.96 & $\mathrm{ab}$ & 7.46 & abcdef & 111.9 & 83.8 \\
\hline 'LP 11-1525/1-3’ & 9.10 & $a b c$ & 9.72 & $\mathrm{ab}$ & 5.84 & bcdefg & 106.8 & 64.2 \\
\hline \multicolumn{9}{|c|}{ Mean } \\
\hline 2 weeks (scale) ${ }^{*}$ & 3.93 & a & 2.41 & $\mathrm{~b}$ & 1.82 & c & $60.6 \pm 13.8$ & $46.6 \pm 7.5$ \\
\hline 4 weeks (scale)* & 4.47 & a & 3.03 & $\mathrm{~b}$ & 2.39 & c & $67.2 \pm 18.3$ & $53.6 \pm 10.2$ \\
\hline 6 weeks $(\mathrm{cm})$ & 9.28 & $\mathrm{a}$ & 7.34 & b & 3.96 & c & $80.2 \pm 31.1$ & $43.6 \pm 21.4$ \\
\hline
\end{tabular}

Different letters indicate statistically significant differences between different treatments. (Duncan's multiple range test; $\mathrm{P}<0.05$ ).

*Level of rooting: scale from 1 (no roots) to 5 (well developed roots)

chemical agents that reduce water potential in the culture medium (Barra et al., 2013). Among these, sorbitol, $\mathrm{NaCl}$, mannitol, agar, polyethylene glycol (PEG) are used. PEG is the most recommended inductor because it does not penetrate plant cells and also reduces the water potential of the medium in which plants develop (Manoj et al., 2011). Data about effects of sorbitol on in vitro potato growth are more limited compared with other agents that induce water stress. Some researchers reported that addition of sorbitol on Murashige-
Skoog medium decreased water potential, inducing drought stress affecting shoot and root growth (Gopal and Iwama, 2007).

The choice of the inducing water stress agents concentration was based on the results obtained by other researchers. Thus, differences in morphological parameters occurred only at $4 \%$ sorbitol, while at $2 \%$ sorbitol plant responses were generally similar to the control level. At 6, 8 and $10 \%$ sorbitol, plants did not produce stems and leaves (Albiski 
Table 5. Effect of sorbitol and PEG on fresh weight of the studied potato genotypes under induced water stress after six weeks of $i$ vitro cultivation

\begin{tabular}{|c|c|c|c|c|c|c|c|c|}
\hline \multirow{3}{*}{$\begin{array}{l}\text { Line (cod) } \\
\text { 'LI 101-3' }\end{array}$} & \multicolumn{6}{|c|}{ Fresh weight $(\mathrm{g})$} & \multicolumn{2}{|c|}{$\begin{array}{l}\text { Relative response compared to the } \\
\text { control (\%) }\end{array}$} \\
\hline & \multicolumn{2}{|c|}{ Control } & \multicolumn{2}{|c|}{ Sorbitol $4 \%$} & \multicolumn{2}{|c|}{ PEG 4.8\% } & \multirow{2}{*}{$\begin{array}{c}\text { Sorbitol 4\% } \\
13.0\end{array}$} & \multirow{2}{*}{$\begin{array}{c}\text { PEG 4.8\% } \\
11.4\end{array}$} \\
\hline & 0.368 & $a b$ & 0.048 & ij & 0.042 & ij & & \\
\hline 'LI 101-6’ & 0.204 & cdefg & 0.058 & hij & 0.050 & ij & 28.4 & 24.5 \\
\hline 'LI 101-7’ & 0.292 & bcd & 0.058 & hij & 0.050 & ij & 19.9 & 17.1 \\
\hline 'LI 102-2' & 0.188 & defgh & 0.016 & j & 0.032 & ij & 8.5 & 17.0 \\
\hline 'LI 102-3' & 0.224 & cdef & 0.084 & ghij & 0.018 & $\mathrm{j}$ & 37.5 & 8.0 \\
\hline 'LI 102-4’ & 0.442 & a & 0.070 & hij & 0.098 & fghij & 15.8 & 22.2 \\
\hline 'LP 11-1525/1-1' & 0.260 & bcde & 0.154 & efghi & 0.118 & fghij & 59.2 & 45.4 \\
\hline 'LP 11-1525/1-2' & 0.262 & bcde & 0.110 & fghij & 0.136 & efghij & 42.0 & 51.9 \\
\hline 'LP 11-1525/1-3' & 0.324 & $\mathrm{bc}$ & 0.156 & efghi & 0.080 & ghij & 48.2 & 24.7 \\
\hline Mean & 0.284 & $\mathbf{a}$ & 0.083 & b & 0.069 & b & $30.3 \pm 17.5$ & $24.7 \pm 14.8$ \\
\hline
\end{tabular}

Different letters indicate statistically significant differences between different treatments (Duncan's multiple range test; $\mathrm{P}<0.05$ )

Table 6. Effect of sorbitol and PEG on dry weight of the studied potato genotypes under induced water stress after six weeks of in vitro cultivation

\begin{tabular}{|c|c|c|c|c|c|c|c|c|}
\hline \multirow{3}{*}{$\begin{array}{l}\text { Line (cod) } \\
\text { 'LI 101-3' }\end{array}$} & \multicolumn{6}{|c|}{ Dry weight (g) } & \multicolumn{2}{|c|}{$\begin{array}{l}\text { Relative response compared to the control } \\
\text { (\%) }\end{array}$} \\
\hline & \multicolumn{2}{|c|}{ Control } & \multicolumn{2}{|c|}{ Sorbitol 4\% } & \multicolumn{2}{|c|}{ PEG 4.8\% } & \multirow{2}{*}{$\begin{array}{c}\text { Sorbitol } 4 \% \\
30.0\end{array}$} & \multirow{2}{*}{$\begin{array}{c}\text { PEG 4.8\% } \\
13.3\end{array}$} \\
\hline & 0.030 & $a b$ & 0.009 & defg & 0.004 & $\mathrm{fg}$ & & \\
\hline 'LI 101-6' & 0.023 & abcde & 0.009 & efg & 0.005 & $\mathrm{fg}$ & 39.1 & 21.7 \\
\hline 'LI 101-7’ & 0.024 & abcde & 0.006 & $\mathrm{fg}$ & 0.006 & $\mathrm{fg}$ & 25.0 & 25.0 \\
\hline 'LI 102-2' & 0.015 & bcdefg & 0.002 & $\mathrm{~g}$ & 0.015 & bcdefg & 13.3 & 100.0 \\
\hline 'LI 102-3' & 0.014 & bcdefg & 0.017 & bcdefg & 0.002 & $\mathrm{~g}$ & 121.4 & 14.3 \\
\hline 'LI 102-4' & 0.029 & $\mathrm{ab}$ & 0.014 & bcdefg & 0.011 & cdefg & 48.3 & 37.9 \\
\hline 'LP 11-1525/1-1' & 0.026 & $\mathrm{abcd}$ & 0.024 & abcde & 0.021 & abcdef & 92.3 & 80.8 \\
\hline 'LP 11-1525/1-2' & 0.026 & $a b c d$ & 0.021 & abcdef & 0.020 & abcdef & 80.8 & 76.9 \\
\hline 'LP 11-1525/1-3' & 0.036 & a & 0.027 & $a b c$ & 0.019 & bcdefg & 75.0 & 52.8 \\
\hline Mean & 0.025 & a & 0.014 & $\mathbf{b}$ & 0.011 & $\mathbf{b}$ & $58.4 \pm 35.9$ & $47.0 \pm 32.2$ \\
\hline
\end{tabular}

Table 7. Pearson correlation of the morphological parameters measured for all time periods

\begin{tabular}{|c|c|c|c|c|c|c|c|c|c|c|c|c|c|}
\hline & \multicolumn{4}{|c|}{2 weeks } & \multicolumn{4}{|c|}{ 4 weeks } & \multicolumn{5}{|c|}{ 6 weeks } \\
\hline & SL2 & NL2 & LA2 & LR2 & SL4 & NL4 & LA4 & LR4 & SL6 & NL6 & LA6 & RL6 & FW6 \\
\hline NL2 & $0.71^{* *}$ & & & & & & & & & & & & \\
\hline LA2 & $0.72^{* *}$ & $0.78^{* *}$ & & & & & & & & & & & \\
\hline LR2 & $0.76^{* *}$ & $0.67^{* *}$ & $0.83^{* *}$ & & & & & & & & & & \\
\hline SL4 & $0.89^{* *}$ & $0.72^{* *}$ & $0.73^{* *}$ & $0.76^{* *}$ & & & & & & & & & \\
\hline NL4 & $0.57^{* *}$ & $0.83^{* *}$ & $0.67^{* *}$ & $0.65^{* *}$ & $0.71^{* *}$ & & & & & & & & \\
\hline LA4 & $0.65^{* *}$ & $0.73^{* *}$ & $0.83^{* *}$ & $0.79^{* *}$ & $0.71^{* *}$ & $0.76^{* *}$ & & & & & & & \\
\hline LR4 & $0.67^{* *}$ & $0.69^{* *}$ & $0.78^{* *}$ & $0.90^{* *}$ & $0.76^{* *}$ & $0.77^{* *}$ & $0.87^{* *}$ & & & & & & \\
\hline SL6 & $0.80^{* *}$ & $0.69^{* *}$ & $0.66^{* *}$ & $0.71^{* *}$ & $0.97^{* *}$ & $0.71^{* *}$ & $0.66^{* *}$ & $0.73^{* *}$ & & & & & \\
\hline NL6 & $0.50^{* *}$ & $0.78^{* *}$ & $0.59^{* *}$ & $0.56^{* *}$ & $0.61^{* *}$ & $0.87^{* *}$ & $0.70^{* *}$ & $0.66^{* *}$ & $0.62^{* *}$ & & & & \\
\hline LA6 & $0.66^{* *}$ & $0.69^{* *}$ & $0.75^{* *}$ & $0.73^{* *}$ & $0.75^{* *}$ & $0.76^{* *}$ & $0.84^{* *}$ & $0.80^{* *}$ & $0.74^{* *}$ & $0.72^{* *}$ & & & \\
\hline RL6 & $0.45^{* *}$ & $0.54^{* *}$ & $0.56^{* *}$ & $0.69^{* *}$ & $0.56^{* *}$ & $0.65^{* *}$ & $0.70^{* *}$ & $0.79^{* *}$ & $0.56^{* *}$ & $0.62^{* *}$ & $0.69^{* *}$ & & \\
\hline FW6 & $0.79^{* *}$ & $0.66^{* *}$ & $0.76^{* *}$ & $0.78^{* *}$ & $0.84^{* *}$ & $0.65^{* *}$ & $0.76^{* *}$ & $0.80^{* *}$ & $0.80^{* *}$ & $0.58^{* *}$ & $0.79^{* *}$ & $0.62^{* *}$ & \\
\hline DW6 & $0.56^{* *}$ & $0.61^{* *}$ & $0.72^{* *}$ & $0.71^{* *}$ & $0.63^{* *}$ & $0.65^{* *}$ & $0.74^{* *}$ & $0.75^{* *}$ & $0.56^{* *}$ & $0.59^{* *}$ & $0.69^{* *}$ & $0.58^{* *}$ & $0.78^{* *}$ \\
\hline
\end{tabular}

** - correlation was significant at $\mathrm{P}=0.01$ level; $\mathrm{N}=135$

SL - stem length (cm); NL - number of leaves; LA - leaf aspect; LR - level of rooting; RL - root length (cm); FW - fresh weight (g); DW - dry weight (g)

et al., 2012). Relating to PEG, a concentration of $4.8 \%$ allowed better discrimination among genotypes than $9.6 \%$ (Barra et al., 2013; Gopal and Iwama, 2007). In several studies (Kosturkova et al., 2008) drought stress was evaluated by using PEG in the following concentrations: 2, 4, 6, 8, 10 and $15 \%$ (w/v). Other researchers have used different amounts $(128,188$ and 235 $\mathrm{g} / \mathrm{L}^{-1}$ ) of PEG6000 to obtain various drought levels (Bahrami $e t$ al., 2012).

Drought leads to decreased tissue water content resulting in inhibited cell elongation (Taiz and Zeiger, 2006). Compared to other species, potato is sensitive to drought because of its shallow root system (Iwama and Yamaguchi, 2006). Drought 
518

slows growth, induces stomatal closure and therefore reduces photosynthesis (Nemeth et al., 2002).

Potato cultivars or clones which are able to maintain relatively high yields at high temperatures have been identified in field trials (Levy, 1984; Malik et al., 1992, cited by Arvin, 2008). In the case of potato, water shortage during the tuberization period reduces yield more than in other development stages (Anithakumari et al., 2011). The major effects of water stress on potato plant are decreases in leaf area and number of leaves, plant height, number of tubers, tuber growth, quality and yield, number of roots and biomass (Tourneux et al., 2003; Schittenhelma et al., 2006; Arvin and Donnelly, 2008; Hassanpanah, 2009).

The present findings suggested that line 'LP 11-1525/1' might be used as potential potato cultivar resistant at drought stress, but further studies and field trials are needed.

\section{Conclusions}

From all the studied potato genotypes, the line IP 11$1525 / 1$ ' achieved good results for most of the growth parameters under study. Lines derived from true potato seeds behaved well, in some cases even exceeding the line derived from meristems. In addition, selection of drought tolerance individuals within populations derived from true potato seeds might be applied for further analyses.

\section{References}

Albiski F, Najla S, Sanoubar R, Alkabani N, Murshed R (2012). In vitro screening of potato lines for drought tolerance. Physiology and Molecular Biology of Plants 18(4):315-321.

Anithakumari AM, Dolstra O, Vosman B, Visser RGF, van der Linden CG (2011). In vitro screening and QTL analysis for drought tolerance in diploid potato. Euphytica 181(3):357-369.

Arvin MJ, Donnelly DJ (2008). Screening potato cultivars and wild species to abiotic stresses using an electrolyte leakage bioassay. Journal of Agricultural Science and Technology 10:33-42.

Bahrami H, Razmjoo J, Ostadi Jafari A (2012). Effect of drought stress on germination and seedling growth of sesame cultivars (Sesamum indicum L.). International Journal of AgriScience 2(5):423-428.

Barra M, Correa J, Salazar E, Sagredo B (2013). Response of potato (Solanum tuberosum L.) germplasm to water stress under in vitro conditions. American Journal of Potato Research 90:591-606.

Bray EA, Bailey-Serres J, Weretilnyk E (2000). Responses to abiotic stresses. In: Gruissem W, Buchnnan B, Jones R (Eds). Biochemistry and molecular biology of plants. American Society of Plant Physiology, Rockville, MD pp 1158-1203.

Cochard H, Barigah S, Kleinhentz M, Eshel A (2008). Is xylem cavitation resistance a relevant criterion for screening drought resistance among Prunus species? Journal of Plant Physiology 165:976-982.

Food and Agriculture Organization of the United Nations (2011). Crops Statistics database. Retrieved 2016 March 12 from http://faostt.fao.org.

Gopal J, Iwama K (2007). In vitro screening of potato against waterstress mediated through sorbitol and polyethylene glycol. Plant Cell Reports 26(5):693-700.
Gopal J, Iwama K, Jitsuyama Y (2008). Effect of water stress mediated through agar on in vitro growth of potato. In Vitro Cellular \& Developmental Biology Plant 44:221-228.

Hassan NM, Serag MS, El-Feky FM (2004). Changes in nitrogen content and protein profiles following in vitro selection of $\mathrm{NaCl}$ resistant mung bean and tomato. Acta Physiologiae Plantarum 26:165-175.

Hassanpanah D (2009). In vitro and in vivo screening of potato cultivars against water stress by polyethylene glycol and potassium humate. Biotechnology, 2009. Asian Network for Scientific Information pp. 1-6.

Iwama K, Yamaguchi J (2006). Abiotic stresses. In: Gopal J, Khurana SM (Eds). Handbook of potato production, improvement and postharvest management. Food Product Press, New York pp 231278.

Jain M, Tiwary S, Gadre R (2010). Sorbitol-induced changes in various growth and biochemical parameters in maize. Plant Soil and Environment 6:263-267.

Khajeh-Hosseini M, Powell AA, Bimgham IJ (2003). The interaction between salinity stress and seed vigor during germination of soybean seeds. Seed Science and Technology 31:715-725.

Kosturkova G, Todorova R, Sakthivelu G, Akitha Devi MK, Giridhar P, Rajasekaran T, Ravishankar GA (2008). Response of bulgarian and indian soybean genotypes to drought and water deficiency in field and laboratory conditions. General and Applied Plant Physiology, special issue 34(3-4):239-250.

Lafitte H, Price A, Courtois B (2004). Yield response to water deficit in an upland rice mapping population: Associations among traits and genetic markers. Theoretical and Applied Genetics 109:1237-1246.

Levy D, Veilleux RE (2007). Adaptation of potato to high temperatures and salinity. American Journal of Potato Research 84:487-506.

Levy D (1984). Cultivated Solanum tuberosum as a source for the selection of cultivars adapted to hot climate. Tropical Agriculture 61:167-170.

Lobell DB, Schlenker W, Costa-Roberts J (2011). Climate trends and global crop production since 1980. Science 333(6042):616-620.

Magombeyi MS, Taigbenu AE (2008). Crop yield risk analysis and mitigation of smallholder farmers at quaternary catchment level: Case study of B72A in Olifants river basin, South Africa. Physics and Chemistry of the Earth 33:744-756.

Malik NJ, Dwelle RB, Thonrton MK, Pavek JJ (1992). Dry matter accumulation in potato clones under seasonal high temperature conditions in Pakistan. American Potato Journal 69:667-676.

Manoj R, Rajwant K, Rohtas S, Manu G, Dhawan AK (2011). Developing stress tolerant plants through in vitro selection - An overview of the recent progress. Environmental and Experimental Botany 71:89-98.

Marron N, Gielen B, Brignolas F, Jian G, Johnson JD, Karnosky DF, Polle A, Scarascia-Mugnozza G, Schroeder WR, Ceulemans R (2008). Poplars and willows in the world, Chapter 7 - Abiotic Stresses. Food and Agriculture Organization of the United Nations, Forestry Department, Working Paper IPC/9-7 FAO, Rome, Italy.

Mittler R (2006). Abiotic stress, the field environment and stress 
combination. Trends in Plant Science 11(1):15-19.

Nemeth M, Janda T, Hovarth E, Paldi E, Szali G (2002). Exogenous salycilic acid increases polyamine content but may decrease drought tolerance in maize. Plant Science 162:569-574.

Noiraud N, Maurousset L, Lemoine R (2001). Transport of polyols in higher plants. Plant Physiology and Biochemistry 39:717-728.

Ober E (2008). Breeding for improved drought tolerance and water use efficiency. Arable cropping in a changing climate. Rothamsted Research, Broom's Barn Research Centre, Higham, Bury St Edmunds pp 28-37.

Pareek A, Sopory SK, Bohnert HJ, Govindjee (2010). Abiotic stress adaptation in plants. Physiological, Molecular and Genomic Foundation.

Rahman MH, Islam R, Haider SA (2008). Differential response of potato under sodium chloride stress conditions in vitro. Journal of Bio-Science 16:79-83.

Rukundo P, Carpentier SC, Swennen R (2012). Development of in vitro technique to screen for drought tolerant banana varieties by sorbitol induced osmotic stress. African Journal of Plant Science 6(15):416-425.
Schittenhelma S, Sourell H, Lopmeierc F (2006). Drought resistance of potato cultivars with contrasting canopy architecture. European Journal of Agronomy 24:193-202.

Taiz L, Zeiger E (2006). Plant physiology, 4th ed. Sinauer Associates Inc, Sunderland, Massachusetts.

Tourneux C, Devaux A, Camacho MR, Mamani P, Ledent JF (2003). Efect of water shortage on six potato genotypes in the highlands of Bolivia (I): morphological parameters; growth and yield. Agronomie 23:169-179.

Vollenweider P, Günthardt-Goerg MS (2005). Diagnosis of abiotic and biotic stress factors using the visible symptoms in foliage. Environmental Pollution 137:455-465.

Zidenga T (2006). Progress in molecular approaches to drought tolerance in crop plants. Information systems for biotechnology. News Repport. 